\section{Concentration of haemodynamic and inflammatory related cytokines in diabetic retinopathy}

\author{
Jl Patel ${ }^{1,2}$, GM Saleh², PG Hykin ${ }^{1}$, ZJ Gregor ${ }^{1}$ and \\ IA Cree ${ }^{2}$
}

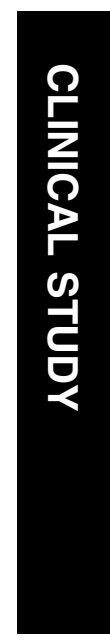

\author{
Abstract \\ Aim There are changes in blood flow during \\ the clinical stages of diabetic retinopathy with \\ increasing leukostasis and secondary \\ elaboration of cytokines. This study evaluated \\ the vitreous concentrations of haemodynamic- \\ related (endothelin-1 (ET-1) and nitric oxide \\ (NO)), inflammatory and anti-inflammatory \\ (interleukin-1 receptor antagonist, IL-1 Ra) \\ cytokines in the diabetic patients (with \\ nonproliferative diabetic retinopathy \\ (NPDR) and proliferative diabetic retinopathy \\ (PDR)), compared them with those of \\ control patients (full thickness macular hole, \\ FTMH) and correlated to macular structural \\ indices. \\ Method Vitreous samples from five FTMH \\ patients representing normal controls \\ were analysed together with the vitreous \\ samples of 15 patients with NPDR and five \\ with PDR. The vitreous concentrations of \\ nitrite (total NO), ET-1, and prostacyclin was \\ determined using ELISA kits (R\&D Systems, \\ Minneapolis, MN, USA) according to the \\ manufacturer's instructions. A sandwich \\ luminescent immunoassay technique was \\ used to determine IL-1 $\beta$ and IL-1 Ra \\ concentrations. \\ Results In the different clinical groups, there \\ were no differences in the vitreous NO and \\ prostacyclin concentrations. In NPDR, the \\ median ET-1 concentration $(0.7 \mathrm{pg} / \mathrm{ml} \mathrm{SD}$ \\ $\pm 0.8 \mathrm{pg} / \mathrm{ml})$ was significantly reduced \\ $(P<0.05)$, compared to PDR $(6.35 \mathrm{pg} / \mathrm{ml} \mathrm{SD}$ \\ $\pm 0.6 \mathrm{pg} / \mathrm{ml})$ and FTMH $(3.6 \mathrm{pg} / \mathrm{ml} \mathrm{SD}$ \\ $\pm 0.14 \mathrm{pg} / \mathrm{ml}$ ). Its concentration also positively \\ correlated with foveal thickness and macular \\ volume $(P<0.05)$ in patients with NPDR and \\ macular oedema. IL-1 $\beta$ was detected in PDR, \\ and diabetic patients demonstrated a lower \\ concentration of the anti-inflammatory \\ cytokine IL-1 Ra.
}

Conclusion Reduced concentrations of ET-1 in NPDR may reflect the haemodynamic changes of NPDR. The IL-1 Ra concentration suggests a change in the anti-inflammatory environment of the diabetic retina. Eye (2008) 22, 223-228; doi:10.1038/sj.eye.6702584; published online 22 September 2006

Keywords: nitric oxide; endothelin-1; prostacyclin; interleukin; macular oedema; diabetes

\section{Introduction}

There are haemodynamic changes in diabetic retinopathy. During disease progression, there is a direct correlation between elevation of blood flow and degree of retinopathy in the advanced stages of nonproliferative retinopathy (NPDR). ${ }^{1}$ This increase in blood flow may subsequently be attenuated with increases in retinal arteriovenous transit time and decreased luminal diameter in proliferative diabetic retinopathy (PDR). ${ }^{2}$

There is also increasing evidence for loss of autoregulation in the diabetic retina. ${ }^{3}$ As autonomic innervation terminates at the lamina cribrosa, retinal blood flow is autoregulated primarily by local factors, and the response of pericytes and endothelial cells to these local factors become altered in chronic hyperglycaemia, disrupting autoregulation. The local factors responsible are primarily nitric oxide (NO) ${ }^{4}$ and endothelin-1 (ET-1). ${ }^{5}$

In addition, multiple studies suggest increased leukocyte adhesion to the retinal capillaries in diabetes secondary to increased activation of leukocytes and monocytes, ${ }^{6}$ with increased expression of adhesion molecules on both these circulating cells and endothelial cells. ${ }^{7}$ As a consequence, local inflammatory
'Department of Pathology, Moorfields Eye Hospital, London, UK

${ }^{2}$ Department of Pathology, Institute of Ophthalmology, London, UK

Correspondence: Jl Patel, Department of Pathology, Institute of Ophthalmology, 11-43 Bath St, London EC1V 9EL, UK Tel: + 447921710875 ; Fax: + 442076086862

E-mail: jigs37@hotmail.com

Received: 24 September 2005

Accepted in revised form: 9 August 2006 Published online: 22 September 2006 
and anti-inflammatory factors may be altered. Previous reports have also suggested the role of prostaglandins in the regulation of vasopermeability and angiogenesis. ${ }^{8}$

This study sought to evaluate the vitreous concentrations of haemodynamic (ET-1 and NO) and inflammatory (interleukin-1 $\beta$ (IL-1 $\beta$ ) and prostacyclin) and anti-inflammatory (interleukin-1 receptor antagonist, IL-1 Ra) markers in primarily NPDR to ascertain if changes in these locally produced factors vary at this stage of retinopathy compared to PDR and also in patients with full thickness macular hole (FTMH).

\section{Patients and methods}

Vitreous samples were collected from patients with idiopathic FTMH, NPDR with clinically significant macular oedema (CSMO) despite previous treatment with conventional ETDRS macular photocoagulation, and PDR. These patients were recruited consecutively from the Surgical Retina Department under the care of ZG at Moorfields Eye Hospital, London, and were to undergo routine pars plana vitrectomy.

Patients with PDR were undergoing vitrectomy for tractional retinal detachment associated with mostly inactive fibrovascular membranes.

Patients with persisting CSMO, which involved the foveal centre at least $1 \mathrm{DD}$ in size for $<2$ years, also had optical coherence tomography (OCT) performed to assess the structural indices of the macula pre- and post-surgery. OCT was employed to give (1) macular profile (single horizontal scan through the foveal centre), (2) foveal thickness, and (3) macular volume calculated from six radial scans centred on fixation and averaged using quantitative assessment software. Exclusion criteria for patients with CSMO were (i) posterior vitreous detachment diagnosed by the presence of a Weiss ring, (ii) macular traction as evidenced by retinal striae involving the foveal center, (iii) macular ischaemia as defined by an enlarged foveolar avascular zone (FAZ $>1000 \mu \mathrm{m}$ ) or significant perifoveal capillary loss on fundus fluorescein angiography (FFA), and (iv) coexistent retinal disease. All CSMO patients underwent standard three-port PPV with elimination and removal of the posterior vitreous cortex, but without peeling of the internal limiting membrane.

All patients were recruited in accordance with the Declaration of Helsinki and with the approval of the Ethics Committee of Moorfields Eye Hospital.

Undiluted vitreous (up to $500 \mu \mathrm{l}$ ) samples were taken immediately at the start of vitrectomy prior to the start of infusion and were immediately placed in ice, centrifuged, and the supernatant divided into aliquots and stored at $-20^{\circ} \mathrm{C}$ until laboratory analysis.

\section{Growth factor analysis}

\section{ELISA for NO, prostacyclin and ET-1}

The vitreal concentration of these growth factors in all three groups of patients was determined using ELISA kits purchased from R\&D Systems, Minneapolis, MN, USA, according to the manufacturer's instructions.

The NO assay determines the total NO, based on the enzymatic conversion of nitrate to nitrite by nitrate reductase. This reaction is then followed by a colorimetric detection of the nitrite as an azo dye product of the Greiss reaction. The Greiss reaction is a two-step diazotization reaction that produces a chromophoric azoderivative that absorbs light at $540-570 \mathrm{~nm}$. The samples were tested in duplicate, as were the nitrate standards, and averaged. NO was detectable in the samples as the minimum concentration was $3.12 \mu \mathrm{mol} / 1$ (intra-assay coefficient of variation was $1.2-5.3 \%$; the inter-assay coefficient of variation was $3.3-7 \%$ ).

The prostacyclin assay relies on determining the concentration of 6-keto- $\mathrm{PGF}_{1 \alpha}$, a nonenzymatic hydration product of prostacyclin $\left(\mathrm{PG}_{2}\right)$. It is a competitive assay where the hydration product competes with a fixed amount of alkaline phosphatase-labelled 6-keto- $\mathrm{PGF}_{1 \alpha}$ for sites on sheep polyclonal antibody. This antibody in turn binds to donkey anti-sheep antibody coated onto the microplate. Following a wash to remove excess conjugate and unbound sample, a substrate solution is added to determine the bound enzyme activity with the absorbance at $405 \mathrm{~nm}$. The intensity of the colour is inversely proportional to the concentration of 6-keto-PGF $\mathrm{Pa}_{1 \mathrm{a}}$ which corresponds to the $\mathrm{PG}_{2}$ concentration. The concentration range of detection was $7.8-2000 \mathrm{pg} / \mathrm{ml}$ and all samples fell within this range (intra-assay coefficient of variation 2.9-9\%; inter-assay coefficient of variation 3.4-9.4\%).

The ET- 1 assay was quantitative sandwich enzyme immunoassay kit (R\&D Systems, catalogue number QET00). A monoclonal antibody specific for ET-1 is coated onto the microplate. Standards and samples are added and any ET- 1 gets bound to the antibody, which is then detected by adding a peroxidase-linked monoclonal antibody to ET-1, which, after further washing, is determined using a luminol-peroxide substrate (LumiGLO Reagent and Peroxide, New England Biolabs, USA) and a luminometer to measure the intensity of the light emitted. The concentration range of detection was $0.32-1000 \mathrm{pg} / \mathrm{ml}$, and all samples fell within this range (intra-assay coefficient of variation $1.5-2.5 \%$; inter-assay coefficient of variation $5.4-10 \%$ ).

\section{ELISA for IL-1 $\beta$ and IL-1 Ra}

The concentrations of these growth factors $(\mathrm{pg} / \mathrm{ml})$ in the three groups of patients were analysed using a sandwich luminescence immunoassay. 
Antibodies against their respective growth factors were used in assays performed in 384-well plates. Primary capture anti-human antibody $(30 \mu \mathrm{l})$ was added to the wells and incubated overnight. All incubations were performed at room temperature $\left(20-25^{\circ} \mathrm{C}\right)$. The wells were blocked with $50 \mu \mathrm{l}$ of phosphate-buffered saline (PBS)- $0.1 \%$ bovine serum albumin (BSA) for $1 \mathrm{~h}$. In total, $30 \mu \mathrm{l}$ of ocular fluid samples were added $(1: 4$ dilution with PBS, $\mathrm{pH} 7.4$ ) and incubated for $2 \mathrm{~h}$. Then, $30 \mu \mathrm{l}$ of the human biotinylated affinity-purified detection or secondary antibody was added followed by the addition of $30 \mu \mathrm{l}$ of Neutravidin Horseradish Peroxidase Conjugate (Pierce, IL, USA) at $0.125 \mu \mathrm{g} / \mathrm{ml}$ for $30 \mathrm{~min}$. Finally, $30 \mu \mathrm{l}$ of LumiGLO Reagent and Peroxide (New England Biolabs, USA) was added for $5 \mathrm{~min}$, and the plate read in a luminometer. Between the addition of each antibody and reactive agent, the wells were washed with PBS, the last wash being with Tris-buffered saline (TBS, $50 \mathrm{mM}, \mathrm{pH}$ 8). All sample readings were quantified from a standard curve produced using recombinant human cytokine (from R\&D).

The nature and the concentrations of each pair of antibody for both growth factors and their respective inter- and intra-assay coefficients of variation $(\mathrm{CoV})$ and concentration sensitivity are shown in Table 1. All antibody pairs and standard growth factor peptides were obtained from R\&D Systems, Minneapolis, MN, USA.

\section{Statistical analysis}

Parametric ANOVA test was used to analyse the different cytokine concentrations between the three groups. Growth factor and macular clinical correlations were assessed using multiple regression analysis. Values are reported as means \pm SD. Two-tailed test results were considered significant at $P<0.05$.

\section{Results}

The average duration of diabetes in the recruited patients was 14 years (5-22 years) with $\mathrm{HbA} 1 \mathrm{C}$ of 9\% (9-12\%), and their average age was 58 (40-72 years). All had type II diabetes. Those patients with moderate to severe nonproliferative retinopathy $(n=15)$ also demonstrated clinically significant macular oedema, which had been present for an average of 15 months (12-18 months) prior to pars plana vitrectomy. They had received on average three macular laser treatments (range 1-6). Those with proliferative disease $(n=5)$ had received extensive pan-retinal photocoagulation and underwent PPV for delamination of membranes and further endolaser. Vitreous samples from five FTMH patients (average age 68 (65-78) years) representing normal control eyes were also analysed.

This sampled vitreous was also being used to ascertain the concentration of other growth factors as part of another study and so where sufficient vitreous was available, a complete set of growth factor analysis of each patient enrolled into this study was performed and in only such circumstances was the correlation analysis with clinical parameters performed.

\section{Vitreous NO (total nitrite)}

The mean concentration in macular-hole patients was $80 \mu \mathrm{mol} / 1$ (SD $\pm 8.5 \mu \mathrm{mol} / \mathrm{l}$ ), while in the NPDR with CSMO group, the mean concentration was $83 \mu \mathrm{mol} / 1$ (SD $\pm 23 \mu \mathrm{mol} / 1$ ) and, in PDR patients, it was $70 \mu \mathrm{mol} /$ $1(\mathrm{SD} \pm 27 \mu \mathrm{mol} / \mathrm{l})$. There were no significant differences in the results between these groups $(P=0.6)$ (Figure 1).

\section{Vitreous prostacyclin}

The mean concentration in macular-hole patients was $50 \mathrm{pg} / \mathrm{ml}$ (SD $\pm 2 \mathrm{pg} / \mathrm{ml}$ ), while in the two diabetic

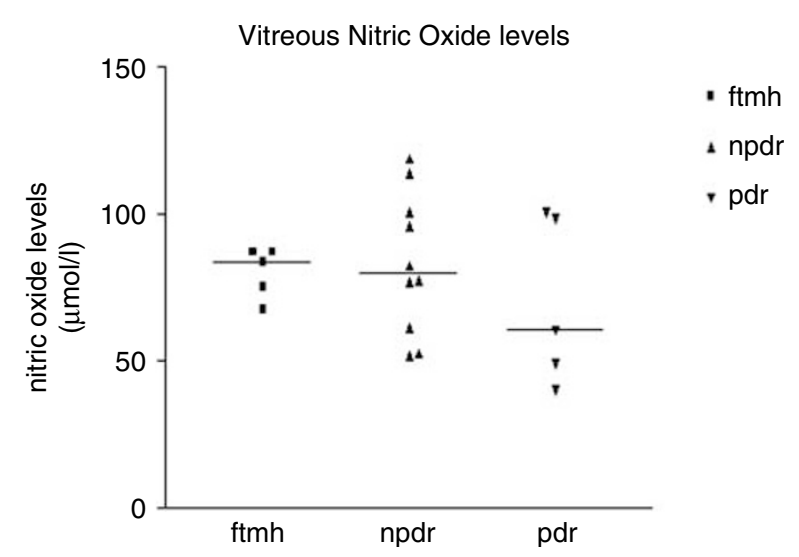

Figure 1 Vitreous NO concentrations in the diabetic retinopathy compared to control. There was no significant difference between the groups.

Table 1 Antibody profile for LIA tests

\begin{tabular}{|c|c|c|c|c|c|}
\hline $\begin{array}{l}\text { RED } \\
\text { antibodies }\end{array}$ & $\begin{array}{l}\text { Primary antibody } \\
\text { concentration }(\mathrm{ng} / \mathrm{ml})\end{array}$ & $\begin{array}{l}\text { Secondary capture } \\
\text { concentration }(\mathrm{ng} / \mathrm{ml})\end{array}$ & $\begin{array}{l}\text { Inter-assay } \\
\operatorname{CoV}(\%)\end{array}$ & $\begin{array}{l}\text { Intra-assay } \\
\operatorname{CoV}(\%)\end{array}$ & Sensitivity \\
\hline IL-1 $\beta$ & Polyclonal, $2000 \mathrm{ng} / \mathrm{ml}$ & Biotinylated, polyclonal, 2 & 17 & 18 & $1 \mathrm{pg} / \mathrm{ml}$ \\
\hline IL-1 Ra & Monoclonal, $2000 \mathrm{ng} / \mathrm{ml}$, clone no. $=10309$ & Biotinylated, polyclonal, $200 \mathrm{ng} / \mathrm{ml}$ & 21 & 21 & $0.5 \mathrm{ng} / \mathrm{ml}$ \\
\hline
\end{tabular}


patient groups the concentrations were largely similar to the macular-hole patients and with each other: $45 \mathrm{pg} / \mathrm{ml}$ in NPDR and PDR (SD $\pm 1 \mathrm{pg} / \mathrm{ml}$ for PDR and $\pm 5 \mathrm{pg}$ / $\mathrm{ml}$ for NPDR with macular oedema). Differences between the groups approached significance, but did not reach it $(P=0.1)$. Diabetic patients had lower concentrations compared to the macular-hole patients representing the normal population (Figure 2).

\section{Vitreous ET-1}

The mean concentration in macular-hole patients was $3.6 \mathrm{pg} / \mathrm{ml}$ (SD $\pm 0.14 \mathrm{pg} / \mathrm{ml}$ ), which decreased in NPDR with CSMO to $0.7 \mathrm{pg} / \mathrm{ml}$ (SD $\pm 0.8 \mathrm{pg} / \mathrm{ml}$ ) but increased in PDR to $6.35 \mathrm{pg} / \mathrm{ml}(\mathrm{SD} \pm 0.6 \mathrm{pg} / \mathrm{ml})$. The difference in concentrations between macular-hole and NPDR groups $(P<0.0001)$ and macular-hole and PDR $(P<0.0001)$ groups was significant as was the difference between the two diabetic groups $(P<0.001)$ (Figure 3$)$. The sensitivity of the assay was $0.32 \mathrm{pg} / \mathrm{ml}$.

\section{Vitreous IL-1 beta}

The concentration in the NPDR with macular oedema patients was below the sensitivity of the assay $(1 \mathrm{pg} / \mathrm{ml})$, but the mean concentration in PDR was $5.58 \mathrm{pg} / \mathrm{ml}$ (SD $\pm 2 \mathrm{pg} / \mathrm{ml}$ ).

\section{Vitreous IL-1 Ra}

The mean concentrations in macular-hole patients was $6.36 \mathrm{ng} / \mathrm{ml}(\mathrm{SD} 3.5 \mathrm{ng} / \mathrm{ml})$ and $3 \mathrm{ng} / \mathrm{ml}(\mathrm{SD} \pm 2.9 \mathrm{ng} / \mathrm{ml})$ in NPDR with macular oedema and $2.7 \mathrm{ng} / \mathrm{ml}$ (SD $\pm 1.7 \mathrm{ng} / \mathrm{ml})$ in PDR. Given the wide variation of

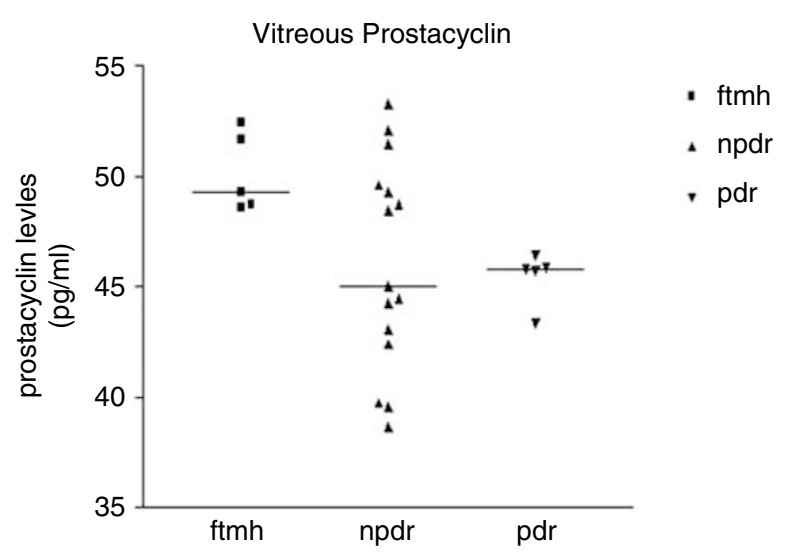

Figure 2 Vitreous prostacyclin concentrations in the diabetic retinopathy compared to control. There was no significant difference between the groups. concentrations in the individual groups, the differences between the groups only approached significance $(P=0.09)$ (Figure 4$)$. (The sensitivity of the assay was $0.5 \mathrm{ng} / \mathrm{ml})$.

\section{Clinical parameters}

OCT in 10 patients with full aqueous growth profile was available.

The median foveal thickness in 10 patients with NPDR with CSMO was $334 \mu \mathrm{m}$ (range 198-751 $\mu \mathrm{m}$ ), while the macular volume was $3.24 \mathrm{~mm}^{3}$ (range $2.13-6.42 \mathrm{~mm}^{3}$ ). In these 10 patients, there was sufficient vitreous for full growth factor analysis and the subsequent regression analysis.

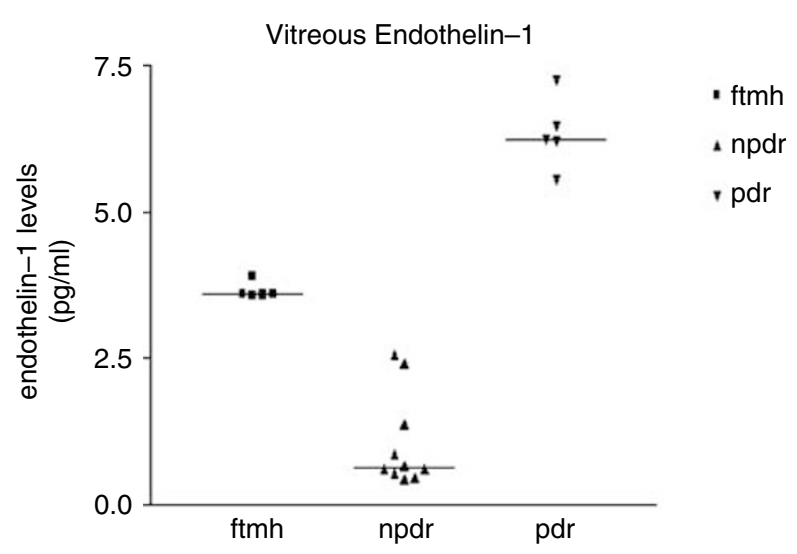

Figure 3 Vitreous ET-1 concentrations in diabetic retinopathy compared to control. There was a significant reduction in concentration in NPDR patients.

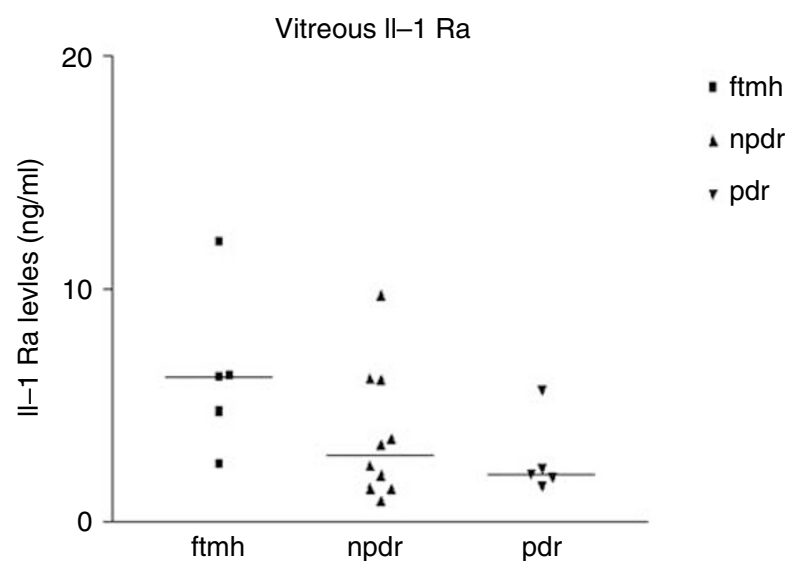

Figure 4 Vitreous IL-1 Ra concentrations in diabetic retinopathy compared to control. There was wide variation in results, with differences between the groups that were not statistically significant. 


\section{Multiple regression analysis}

On performing this analysis, using the clinical parameters as described above, only ET-1 levels demonstrated a relationship with the macular structural indices: with foveal thickness $\left(R^{2}=72 \%, P=0.04\right)$ and with macular volume $\left(R^{2}=75 \%, P=0.03\right)$, possibly suggesting the greater the foveal thickness or macular volume, the higher the vitreous ET-1 concentrations in patients with NPDR and macular oedema.

\section{Discussion}

The retinal microcirculation undergoes changes even before the onset of clinical disease. In type 1 diabetes, there is a glycaemic-control-related increase in capillary pressure and flow resulting from injury to the endothelial cell that stimulates an increase in extracellular matrix production. This leads to basement membrane thickening and disturbed autoregulation. ${ }^{9}$ In type 2 diabetes, there is an altered microvascular vasodilatory capacity. Both situations lead to an increase in capillary perfusion. ${ }^{10}$ According to Starling's principle, an increase in capillary pressure would induce increased passage of fluid and macromolecules into the retinal interstitium.

Our results demonstrate that in diabetes mellitus there are changes in haemodynamic-related, inflammatory, and anti-inflammatory factors. However, concentrations of these factors varied widely even in similar clinically defined patient groups, perhaps suggesting a variation in disease activity between patients.

ET-1 showed decreases in NPDR compared to controls with a subsequent increase in proliferative retinopathy. The reduction in ET-1 in NPDR could reflect the increase in retinal blood flow seen in NPDR as its vasoconstrictive effect would be minimal. Also in nonproliferative patients with macular oedema, our results demonstrated a positive correlation between ET- 1 and both the foveal thickness and the macular volume. A possible mechanism to account for this is that ET-1 can interact with endothelin A and B 1 and B 2 receptors. The endothelin A receptor mediates vasoconstriction, while the $\mathrm{B} 1$ receptor mediates vasodilatation via the release of $\mathrm{NO}$, and the B 2 receptor mediates vasoconstriction. ${ }^{11}$ The correlation of macular structural indices of oedema with ET-1 in the NPDR patients could be related to the activation of ET-B1 receptor producing vasodilatation, while in PDR patients who have greater levels of ET-1, which would allow for a vasoconstrictive effect via the activation of both endothelin receptors A and B 2. Raised levels of ET-1 have also been reported in the vitreous of PDR patients. ${ }^{12}$

Previous reports have shown increased levels of nitrate and nitrite (NO) in PDR. ${ }^{12-14}$ Our results suggest that diabetic patients show no significant differences from controls in their vitreal NO concentrations, even though the diabetic patients did have lower levels compared to controls. These differences in results may be related to the method of analysing NO levels and the nature of NO itself. Our method used an ELISA kit detecting total nitrite levels as an azo dye product of the Greiss reaction. Nitrite is produced from nitrate, which itself is the product of conversion of NO with oxygen. Others have measured nitrate or nitrite levels in vitreous or aqueous using liquid chromatography or spectrophotometry. These differences may reflect the difficulty in measuring this short-acting molecule and of relying on indirect measurements of its subsequent metabolites to reflect its concentration. Despite this discrepancy in the results of NO (ours lower, previous reports higher), it still suggests that there is an abnormal change in its concentration in diabetic retinopathy, altering the vasoregulatory capacity of retinal vessels. However, it may not be the sole mediator for altering or producing the reduced retinal flow seen in PDR.

IL-1 $\beta$ concentrations were raised in PDR patients, while in NPDR and in controls, the levels were below that of sensitivity of the assay. This may suggest that in the early stages of retinopathy, there is minimal acute inflammatory activity, but as disease progresses with significant capillary loss and retinal ischaemia leading to PDR, inflammation increases. Aligned with this change, the anti-inflammatory cytokine IL-1 Ra was significantly higher in the control vitreous compared to the diabetic vitreous which, therefore, may allow for an unhindered activity of the proinflammatory cytokines as disease progresses to PDR.

A recent report has suggested that even in the presence of leukostasis impacting upon multiple retinal capillaries during the early stages of retinopathy, retinal blood flow is not affected. ${ }^{15}$ Therefore, as disease progresses to PDR, the gradual increase in leukostasis is possibly unlikely to produce the decrease in retinal blood flow seen in PDR. The leukostasis itself seems to develop secondary to endothelial dysfunction induced by elevated free fatty acids produced as a metabolic consequence of insulin resistance. ${ }^{15}$ As disease progresses, a greater level of leukostasis maybe seen as endothelial dysfunction increases throughout the retina. This would allow the activated leukocytes to elaborate a host of inflammatory cytokines, for example, IL-1 $\beta$, which is present at high levels in PDR.

Prostacyclin concentrations reflected NO levels. In diabetic patients, the levels were lower than in controls, but not significant. Reports have suggested that NO can stimulate prostacyclin production by the endothelial cell, ${ }^{16}$ and our results for both factors may reflect this finding. 
This is the first report demonstrating the ET-1 concentration in nonproliferative retinopathy and proliferative diabetic retinopathy. They suggest a decrease in ET-1 in NPDR reflecting the increase in retinal blood flow in NPDR with a subsequent increase in ET-1 in PDR, reflecting the attenuation of blood flow in PDR. Furthermore, in the data describing the concentrations of the various markers of inflammation and anti-inflammation, there is a suggestion that the anti-inflammatory environment in diabetes mellitus is reduced compared to controls.

\section{References}

1 Clermont A, Aiello L, Mori F, Bursell S. Vascular endothelial growth factor and severity of nonproliferative diabetic retinopathy mediate retinal hemodynamics in vivo: a potential role for vascular endothelial growth factor in the progression of nonproliferative diabetic retinopathy. Am J Ophthalmol 1997; 124: 433-446.

2 Kohner E, Hamilton A, Saunders S, Sutcliffe B, Bulpitt C. The retinal blood flow in diabetes. Diabetologia 1975; 11: 27-33.

3 Grunwald J, Riva C, Brucker A, Sinclair S, Petrig B. Altered retinal vascular response to $100 \%$ oxygen breathing in diabetes mellitus. Ophthalmology 1984; 91: 1447-1452.

4 Schmetterer L, Salomon A, Rheinberger A, Unfried C, Lexer F, Wolzt M. Fundus pulsation measurements in diabetic retinopathy. Graefes Arch Clin Exp Ophthalmol 1997; 235: 283-287.

5 de la Rubia G, Oliver F, Inoguchi T, King G. Induction of resistance to endothelin-1's biochemical actions by elevated glucose levels in retinal pericytes. Diabetes 1992; 41: 1533-1539.

6 Miyamoto K, Ogura Y. Pathogenetic potential of leukocytes in diabetic retinopathy. Semin Ophthalmol 1999; 14: 233-239.
7 Miyamoto K, Khosrof S, Bursell S, Rohan R, Murata T, Clermont A et al. Prevention of leukostasis and vascular leakage in streptozotocin-induced diabetic retinopathy via intercellular adhesion molecule-1 inhibition. Proc Natl Acad Sci USA 1999; 96: 10836-10841.

8 Fujii E, Irie K, Ogawa A, Ohab K, Muraki T. Role of nitric oxide and prostaglandins in lipopolysaccharide-induced increase in vascular permeability in mouse skin. Eur J Pharmacol 1996; 297: 257-263.

9 Tooke J. Microvascular haemodynamics in diabetes mellitus. Clin Sci 1986; 70: 119-125.

10 Sandeman D, Pym C, Green E, Shore A, Tooke J. Profound impairement of microvascular vasodilatation in the feet of newly diagnosed non-insulin dependent diabetes (NIDDM). Diabetic Med 1990; 7: A25.

11 Rubanyi G, Polokoff M. Endothelins: molecular biology, biochemistry, pharmacology, physiology and pathophysiology. Pharmacol Rev 1994; 46: $325-415$

12 Oku H, Kida T, Sugiyama T, Hamada J, Sato B, Ikeda T. Possible involvement of endothelin-1 and nitric oxide in the pathogenesis of proliferative retinopathy. Retina 2001; 21: 647-651.

13 Hattenbach L, Allers A, Klais C, Koch F, Hecker M. L-arginine-nitric oxide pathway-related metabolites in the aqueous humour of diabetic patients. Invest Ophthalmol Vis Sci 2000; 41: 213-217.

14 Yilmaz G, Esser P, Kociek N, Aydin P, Heimann K. Elevated vitreous nitric oxide levels in patients with proliferative diabetic retinopathy. Am J Ophthalmol 2000; 130: 87-90.

15 Abiko T, Abiko A, Clermont A, Shoelson B, Horio N, Takahasi J. Characterization of retinal leukostasis and hemodyanmics in insulin resistance and diabetes. Diabetes 2003; 52: 829-837.

16 Davidge S, Baker P, McLauglin M, Roberts J. Nitric oxide produced by endothelila cells increases production of eicosanoids through activation of prostaglandin $\mathrm{H}$ synthase. Circ Res 1995; 77: 247-284. 